\title{
Detection of mixed infection of Plasmodium species in the Southern province of Rwanda. Case study: Ruhango, Bunyogombe and Kibilizi Health centres
}

\author{
Regis Ndahiro a, Pascal Bizimana ${ }^{a}$ Ella Larissa Ndoricyimpaye ${ }^{a}$, Alphonse Hakizimana ${ }^{\text {, Jean }}$ \\ Damascene Mfizia , Honore Minani² \\ University of Rwanda- College of Medicine and Health Sciences-Department of Biomedical Laboratory Sciences \\ Institute of Applied Sciences -INES Ruhengeli \\ *Correspondence to Ella Larissa Ndoli (ellalarissan@gmail.com
}

\begin{abstract}
Background: Malaria is a leading cause of mortality and morbidity in sub-Saharan Africa including Rwanda. Though the prevalence of malaria has been reduced due to the use of indoor residual sprayings and insecticide-treated bed nets, it is still a disease that kills many people annually. Many studies conducted revealed that in sub-Saharan Africa including Rwanda there is a high prevalence of Plasmodium falciparum. However, there is still a gap in the identification of the presence of mixed Plasmodium infection. This study was conducted to determine the overall prevalence of Plasmodium species as well as that of mixed plasmodium infection in Ruhango and Kibilizi Health centres.

Methods: Descriptive cross-sectional study was conducted on 126 participants in Ruhango, Bunyogombe and Kibilizi health centres located in the southern province of Rwanda. The conventional sampling strategy was used for the selection of individuals who consented to participate in the study. Blood samples were used to detect Plasmodium species and the obtained data were analyzed using Microsoft excel and IBM SPSS version 21

Results: Among 126 participants presenting with signs and symptoms of malaria, the overall positive cases of Plasmodium species were $61(48.4 \%)$ and among the total positive cases $56(44.5 \%)$ were infected with single Plasmodium species while $5(4 \%)$ were infected with mixed Plasmodium species. Plasmodium falciparum was the most prevalent species infecting 49 (39\%) participants while Plasmodium vivax was the least prevalent infection, detected in only $1(0.8 \%)$ participant.

Conclusion: The study identified the significant prevalence of mixed-species of Plasmodium infection as well as the high prevalence of Plasmodium falciparum infection in the study population. These findings suggest that there is a need for continued monitoring of nonffalciparum infection in this population and the introduction of species-specific RDTs that can be used for diagnostic purposes.
\end{abstract}

Keywords: Malaria, Plasmodium species, Mixed Plasmodium infection, Infection, Prevalence, Descriptive Cross-sectional study, Samples, Purposive sampling, WHO.

\section{INTRODUCTION}

$\mathrm{M}$

alaria is a human disease caused by five Plasmodium parasites: Plasmodium falciparum,

$P$. vivax, $P$. malariae, $P$. ovale and $P$. knowlesi. Plasmodium species are differently distributed in the world. ${ }^{1}$ The prevalence of malaria cases and deaths differs during different seasons. ${ }^{2}$ In 2017, there were an estimated 219 million cases of malaria in 90 countries. Malaria deaths reached 435000 in 2017.3 The World Health Organisation (WHO) African Region carries a disproportionately high share of the global malaria burden in 2017 as well as in 2018. In 2017, the region was home to $92 \%$ of malaria cases and $93 \%$ of malaria deaths ${ }^{3}$ while In 2018, malaria cases were $93 \%$ and $94 \%$ of malaria deaths. ${ }^{4}$

In Africa, including Rwanda, many epidemiological studies demonstrate that $\mathrm{P}$. falciparum is the most prevalent malarial species. ${ }^{5,6}$ Some population groups are at considerably higher risk of contracting malaria and developing severe disease than others. ${ }^{7}$ These include infants, children under five years of age, 
pregnant women and patient with HIV AIDS as well as non-immune migrants, mobile populations and travelers. ${ }^{8}$ In Rwanda, the Malaria indicator survey (MIS) indicated that in 2017 the prevalence of Malaria in the general population was $7 \%$ where Eastern province presented $12 \%$, Southern province $9 \%$, Western province $2 \%$, Northern $1 \%$, and Kigali city $3 \%$. ${ }^{9 a}$ The Rwandan National Malaria control programmes have been at the forefront of scaling up malaria interventions over the past decade, including the distribution of insecticide-treated bed nets, indoor residual spraying in high malaria transmission foci and epidemic-prone areas, early adoption of artemisininbased combined therapy and use of rapid diagnostic test (RDTs) for clinical management of malaria. ${ }^{\mathrm{b}}$

Rapid and accurate diagnosis of Plasmodium infections is crucial for morbidity and mortality reduction in tropical areas, especially in regions where mixed infections are prevalent such as sub-Saharan Africa including Rwanda, where all four parasites infecting humans coexist and mixed-species infections are common and not well characterized but may play roles in disease progression and outcomes. ${ }^{10}$ For improving accuracy in large epidemiological studies, molecular diagnostic tools permitting high throughput analysis for the identification and quantification of malaria parasites would be of great benefit. $^{11}$ Traditionally, light microscopy (LM) examination of blood smears has been considered the gold standard for the diagnosis of malaria. ${ }^{8}$ Light Microscopy has clear advantages over molecular typing since it incurs only low costs, does neither need complex sample preparation nor advanced technology and permits species identification and quantification. ${ }^{8}$ In Rwanda, the use of Rapid diagnostic tests (RDT) by community health workers has greatly facilitated the diagnosis of malaria disease in remote areas where Plasmodium parasites are known to be endemic. ${ }^{12}$ However, their use is limited due to the lack of sensitivity to differentiate between existing Plasmodium species. ${ }^{8}$

For improving accuracy in diagnosis and treatment of malaria, identifying malaria species and or mixed species of Plasmodium infections are reliable methods to move towards the elimination of Plasmodium parasite..$^{5}$ It follows that further understanding of the distribution of local malaria species and co-infections of plasmodium parasites is important for developing appropriate preventive as well as diagnostic and treatment options. This study aimed to determine the prevalence of Plasmodium species co-infection in Ruhango, Bunyogombe Kibilizi health centres. This information is necessary to guide and inform management and control strategies as wells as provide evidence for strategic malaria diagnostics service choices and treatment options.

Fisher's exact test for proportions, were used to test for independence.

\section{METHODOLOGY}

Design and Study area

This was a descriptive cross-sectional study conducted during the dry season for a period of three months from May to September 2019 at Ruhango Health Center of Ruhango district and Kibilizi Health Center of Gisagara district located in the southern province of Rwanda. The above districts are known to be the hot spot zone of malaria disease in Rwanda.

\section{Sample size determination and Sampling}

A purposive sampling strategy was used to select the participants attending Ruhango and Kibilizi health centres presenting signs and symptoms of malaria. Sample size calculation used the prevalence of malaria in the southern province in 2017 which was $9 \%$ as indicated by Rwanda Malaria indicator survey. ${ }^{9}$ The minimum sample size was calculated using Daniel's formula which is $n=\frac{z^{2} * p(1-p)}{d^{2}}$,

where $\mathrm{n}=$ sample size, $\mathrm{p}=$ expected prevalence, $\mathrm{d}=$ allowed error which is $5 \%$, and $\mathrm{z}$ which is statistical of confidence $95 \% .^{13}$

The sample size was: $n=\frac{z^{2} * p(1-p)}{d^{2}}=\frac{1.96^{2} * 0.09(1-0.09)}{0.05^{2}}=$ 126

\section{Data collection and Diagnostic procedures}

Finger prick was performed to prepare thick and thin smears of 1 to $1 \mathrm{~cm}$ and allow the prepared smears to air dry. The well-dried smears were stained with $10 \%$ Giemsa solution for 20 minutes and examined under a light microscope using a 100x objective (immersion oil objective) for detecting the presence of the Plasmodium parasites. Once the parasites were detected on a thick smear using a light microscope, the thin smear was performed for differentiation of Plasmodium species after fixing the smear in methanol and staining it using Giemsa solution diluted $10 \%$ for 20 min and examine under the light microscope using 100x objective for species identification. Slides were considered as positive only after being confirmed by two more technicians. Parasite density was determined as the number of parasites per 200 leukocytes count of $8000 / \mu \mathrm{l}$ of blood. A negative result was considered if no parasites were detected per 200 leukocytes. The four technicians for microscopy detection were well trained on malaria diagnosis, blood collection, thick and thin blood smear preparation, Giemsa staining and using slide reader.

\section{Data record and analysis}

The data were recorded in Microsoft excel and were analyzed using IBM SPSS version 21 (Chicago- USA). The Mantel Haenszel chi-square test, and in some cases

Ethical Approval 
Ethical clearance for the study was obtained from the Institutional Review Board of the College of Medicine and Health Sciences at the University of Rwanda (CMHS/IRB/216/2019). The ethical clearance was presented to study sites administration at Ruhango and Kibilizi Health Centres and an approval letter for the data collection was provided. The community at the study sites was sensitized and appreciated the objectives of the study. Informed consent forms for data collection were signed and the participant's information was kept confidential. Included in the study were the residents of the study site for 6 months preceding the study with clinical signs and symptoms of malaria. Those excluded were nonresidents, those on anti-malarial treatment and those who declined to give informed consent.

\section{RESULTS}

This study was carried out in the southern province, at Ruhango and Kibilizi Health Centers for a period of 3 months from May to September 2019. The study aimed to detect the mixed infection of Plasmodium species using microscopy technique.

\section{Socio-Demographic Characteristics of the study population \\ One hundred and twenty-six participants aged between 3 and 67 years old from Ruhango, Bunyogombe and Kibilizi health centres have participated in the study (Table 1 ).}

TABLE 1: Socio Demographic Characteristics of the Study Population

\begin{tabular}{cccccc}
\hline \multicolumn{5}{c}{ Health Facility } \\
\hline Characteristics & Ruhango & Bunyogombe & Kibilizi & Total (\%) \\
\hline Sex & Male & 37 & 8 & 6 & $51(40.5)$ \\
& Female & 58 & 11 & 6 & $75(59.5)$ \\
& Total & 95 & 19 & 12 & 126 \\
& \multicolumn{1}{c}{ Age } & 28 & 3 & 2 & $33(26.2)$ \\
& $10-20$ & 16 & 6 & 3 & $25(19.8)$ \\
& $21-30$ & 26 & 4 & 1 & $31(24.6)$ \\
& $11-40$ & 11 & 1 & 1 & $13(10.3)$ \\
& $81-50$ & 8 & 1 & 3 & $12(9.5)$ \\
& Total & 6 & 4 & 2 & $12(9.5)$ \\
\hline
\end{tabular}

The most affected population aged $<10$ years old and represent $26.2 \%(33 / 126)$ of the total participants. In this study, $75(59.5 \%)$ were females and $51(40.5 \%)$ were males. All the participants were from Southern province especially at Ruhango Health Center, Buntogombe Health Post and Kibirizi Health Center.

Prevalence of Plasmodium species infection in Ruhango, Kibilizi, and Bunyogombe health centres. As summarized in Figure 1, among the 126 persons who participated in the study, $61(48.4 \%)$ were positive for plasmodium infection and $65(51.6 \%)$ were negative for Plasmodium infection. Among the total positive cases, $56(44.5 \%)$ were infected by single species of plasmodium while $5(4 \%)$ were mixed plasmodium species infections. The most prevalent Plasmodium species was Plasmodium falciparum with 49(39\%) and the least prevalent species was Plasmodium vivax with $1(0.8 \%)$. The prevalence of Plasmodium species per health facility are detailed in the Table 2 below.

Prevalence of mixed infections of plasmodium species As shown in Table 2, out of 126 participants who participated in the study, $5(4 \%)$ had mixed Plasmodium infection. $3(2.4 \%)$ were infected with both Plasmodium falciparum and Plasmodium ovale while $2(1.6 \%)$ were infected with both Plasmodium falciparum and Plasmodium malariae. 


\section{FIGURE 1: Prevalence of Plasmodium species Infection in Ruhango, Kibilizi, and Bunyogombe Health Centers}

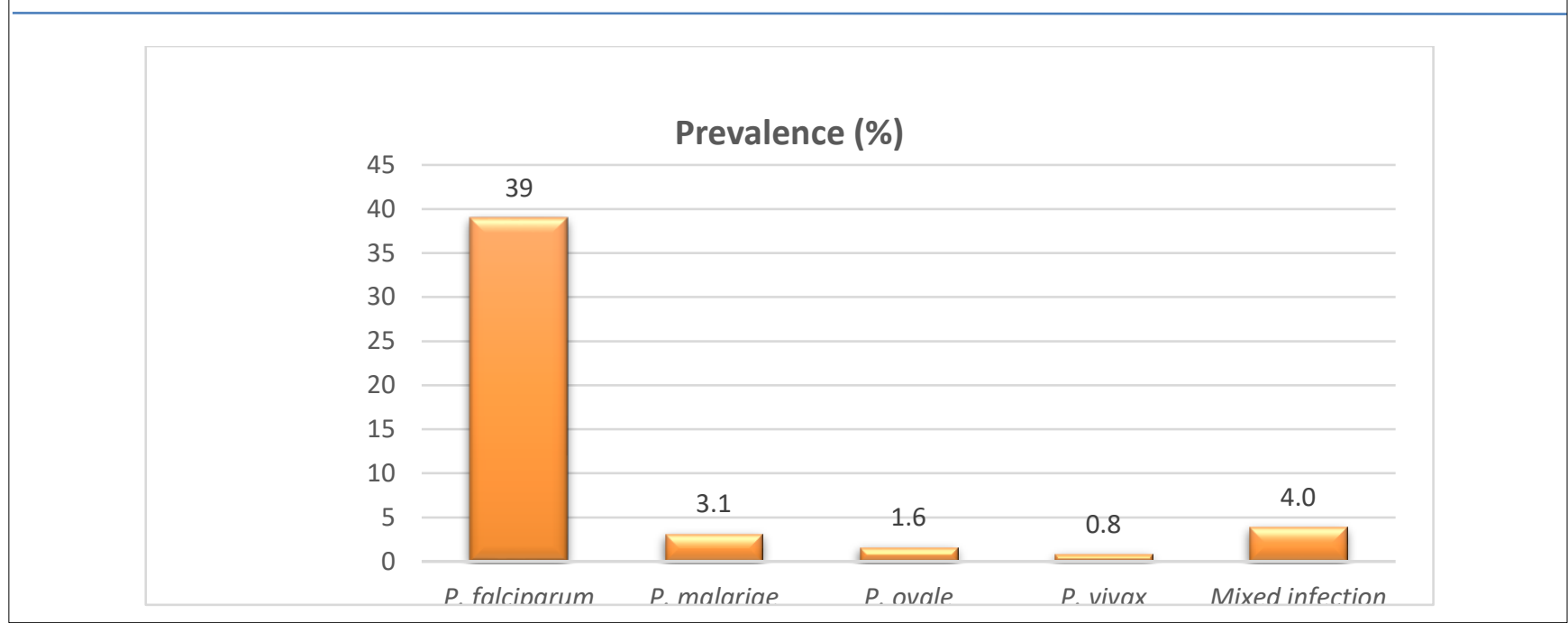

TABLE 2: Socio Demographic Characteristics of the Study Population

\begin{tabular}{lccccc}
\hline \multirow{2}{*}{$\begin{array}{c}\text { Health } \\
\text { facilities }\end{array}$} & $\begin{array}{c}\text { Number } \\
\text { of } \\
\text { samples }\end{array}$ & $\begin{array}{c}\text { P. Falciparum \& P. } \\
\text { ovale }\end{array}$ & $\begin{array}{c}\text { P. Falciparum } 8 \\
\text { P. Malariae }\end{array}$ & Total & P Value \\
\hline Ruhango & 95 & $2(1.6 \%)$ & $1(0.8 \%)$ & $3(2.4 \%)$ & .004 \\
Bunyogombe & 19 & $1(0.8 \%)$ & $1(0.8 \%)$ & $2(1.6 \%)$ & $<.001$ \\
Kibilizi & 12 & $0(0 \%)$ & $0(0 \%)$ & $5(0 \%)$ & $5(4 \%)$ \\
Total & 126 & $3(2.4 \%)$ & $2(1.6 \%)$ & & \\
\hline
\end{tabular}

\section{DISCUSSIONS}

This study showed a significantly high prevalence of Plasmodium species in Ruhango, Bunyogombe and Kibilizi health centres located in the southern province. The overall prevalence was $48.4 \%$. Plasmodium falciparum presented a high prevalence of $39 \%$. Other Plasmodium species such as Plasmodium malariae had $3.1 \%$, Plasmodium ovale $1.6 \%$ and Plasmodium vivax $0.8 \%$. The study demonstrated that mixed infections account for $5 \%$ of the prevalence of the total infections. The prevalence of mixed Plasmodium infection in this study is not different from a study conducted in South Korea which indicated a $2.1 \%$ prevalence of mixed Plasmodium falciparum and Plasmodium ovale coinfection among Korean individuals who had returned from the Democratic Republic of Congo suggesting that in the region of sub-Saharan Africa there is a significant prevalence of mixed Plasmodium infection which are often unrecognized. ${ }^{16}$ Ruhango health centre had a high percentage of positives cases $(37.3 \%)$ and had a higher prevalence of Plasmodium species compared to Kibilizi health center located in the Gisagara District. These findings suggest that there is a need for continued monitoring of non-falciparum infection in this population to decide when the species-specific RDTs can be introduced for diagnostic purposes as the country moves towards malaria elimination. In addition, ACTs, the first-line treatment does not clear plasmodium hypnozoites and radical treatment with primaquine, effective against hypnozoites may play an important role in the control and eventual elimination of P. vivax, $\mathrm{P}$ ovale and $\mathrm{P}$. malariae. ${ }^{15}$ The high prevalence may be attributed to the fact there was no use of indoor residual sprayings at the time of data collection which could reduce malaria vectors and the transmission rate. This prevalence of Plasmodium species in Ruhango and Kibilizi health centres is significantly higher compared to other studies conducted by Gahutu et al, 2011 and 
Nyirakanani et al, 2018 with a prevalence of $16.7 \%$ and $12.2 \%$, respectively. ${ }^{14}$ The patterns of the burden of infection observed for the different species were not surprising as similar observations have been reported elsewhere. ${ }^{17,18}$ However, the fact that the nonfalciparum malaria cases were present in the study population necessitates the need to have tools at the national level that facilitate the detections of nonfalciparum parasites. Also there is a need to conduct refresher courses for laboratory staff and microscopists to strengthen non-falciparum malaria diagnosis. Therefore, future effective malaria control requires an elaborate understanding of the interactions among different plasmodium species.

\section{LIMITATIONS}

This study was conducted in Ruhango and Kibilizi Health centres located in Ruhango and Gisagara districts, respectively and the outcome of the two Health centres cannot be applied to the whole country. Further research is needed to establish the exact prevalence of mixed Plasmodium infection in the whole country.

\section{CONCLUSION}

This study revealed the presence of mixed infections in the Southern province of Rwanda with a prevalence of $5 \%$. The overall prevalence of Plasmodium species was $48.4 \%$ where $P$. falciparum is the most prevalent species with $39 \%$ prevalence. This observation calls for improvement of existing malaria control strategies at the National level. The presence of non-falciparum infections varies with transmission patterns, demographic trends and geographical contrasts. There is a need to monitor those factors through a functional surveillance system to understand the epidemiological profile. Given that most of the mixed infections cannot be detected by HRP2- based RDTs there is a need to strengthen the detection of malaria infection with microscopy examination in Health care facilities to facilitate the detection of non-falciparum infections. Nonetheless, there is a need for further studies to generate a detailed profile and epidemiology of plasmodium species in the country.

\section{REFERENCES}

1. Zeb J, Khan MS, Ullah N, Ullah H and Nabi G. Epidemiology of Plasmodium Species and Prevalence of Malaria based on Age, Sex, Area, Seasonality and Clinical Manifestation in the Population of District Lower Dir, Khyber Pakhtunkhwa, Pakistan. World Journal of Zoology, 2015; 10 (2): 147-152. DOI:10.5829/idosi.wjz.2015.10.2.93158

2. Teshiwal Deress and Mekonnen Girma. Plasmodium falciparum and Plasmodium vivax Prevalence in Ethiopia: A Systematic Review and Meta-Analysis. Malar Res Treat. 2019; 2019:7065064. DOI:10.1155/2019/7065064
3. World Health Organization: World Malaria Report 2018. https://www.who.int/malaria/publications/world-malariareport-2018/en/pdf. Published 19 November 2018. Accessed March 20, 2020.

4. World Health Organization: World Malaria Report 2019. https://www.who.int/publications/i/item/9789241565721. Published December 4, 2019. Accessed March 20, 2020.

5. Bhatt S, Weiss D.J, Cameron E et al. The effect of malaria control on Plasmodium falciparum in Africa between 2000 and 2015. Nature, 2019; 526(7572):207-211. DOI: 10.1038 /nature 15535 .

6. Munyekenye OG, Githeko AK, Zhou G, Mushinzimana E. Plasmodium falciparum Spatial Analysis, Western Kenya Highlands. Emerg Infect Dis. 2005; 11(10):1571-1577. DOI: 10.3201 /eid 1110.050106 .

7. Bizimana J, Twarabamenye E, Kienberger S. Assessing the social vulnerability to malaria in Rwanda. 2015. Malar J, 2015; 14:2. DOI: $10.1186 / 1475-2875-14-2$.

8. Pembele GN, Rivero LR, Fraga J. Detection and Species Identification of Malaria Parasites by Nested-PCR : Comparison with Light Microscopy and with SD BIOLINE Malaria Ag Test in Luanda, Angola. International Journal of TROPICAL DISEASE $\quad$ G $\quad$ Health, 10(1), 1-13. https://doi.org/10.9734/IJTDH/2015/18744.

9. Rwanda Ministry of Health: Malaria indicator Survey 2017. https://dhsprogram.com/pubs/pdf/MIS30/MIS30.pdf. Published in July 2018. Accessed on January 10, 2020.

10. Snow RW, Sartorius B, Kyalo D, et al. The prevalence of Plasmodium falciparum in sub-Saharan Africa since 1900. Nature. 2017:550(7677):515-518. doi:10.1038/nature24059.

11. Mehrotra RK, Lorry K, Kastens W, et al. Random Distribution Of Mixed Species Malaria Infections In Papua New Guinea. Am J Trop Med Hyg.2000; 62(2):225-231. DOI: 10.4269/ajtmh.2000.62.225.

12. Asingizwe D, Rulisa S, Asiimwe-Kateera B, Kim MJ. Malaria elimination practices in rural community residents in Rwanda : A cross-sectional study. Rwanda Journal Series F: Medicine and Health Sciences 2015;2(1):53-59. DOI: 10.4314/rjhs.v2il.7F.

13. Naing L, Winn T, Rusli BN. Practical Issues in Calculating the Sample Size for Prevalence Studies. Archives of Orofacial Sciences 2006: 1: 9-14.

14. Nyirakanani C, Chibvongodze R, Habtu M, Masika M, Mukoko D, Njunwa KJ. Prevalence and risk factors of asymptomatic malaria among under-five children in Huye District, Southern Rwanda. Tanzania Journal of Health Research, 20(1):1-7. https://doi.org/10.4314/thrb.v20i1.

15. Gahutu J, Steininger C, Shyirambere C, Zeile I, Cwinya-ay N. Prevalence and risk factors of malaria among children in southern highland Rwanda. Malar J. 2011;10(1):134. DOI:10.1186/1475-2875-10-134.

16. Kim G, Hong H, Kim SY, et al. Mixed Infection with Plasmodium falciparum and Plasmodium ovale in a Returned Traveller: the First Case in Korea. J Korean Med Sci. 2019; 34(3):1-5. DOI: 10.3346/jkms.2019.34.e23.

17. Zakeri S, Karak Q, Ghasemi F et al. Detection of mixed Plasmodium falciparum infection $\& P$. vivax infections by Nested PCR in Pakistan, Iran \& Afghanistan. Indian J Med Res. 2010;132:31-35.

18. Sitali L, Chipeta J, Miller JM, et al. Patterns of mixed Plasmodium species infections among children six years and under in selected malaria hyper-endemic communities of Zambia: population-based survey observations. BMC Infect Dis. 2015;15:204. doi:10.1186/s12879-015-0935-7

\section{Peer Reviewed}

Competing Interests: None declared.

Received: 10/20/2020; Accepted: 3/8/2021

Cite this article as: Ndahiro R, Bizimana P, Ndoricyimpaye El, Hakizimana A, Mfizi Jd. Mixed infection of Plasmodium species in Southern Rwanda. E Afr Sci. 2021;3(1):112-117. http://doi.org/10.24248/EASci-D-20-00015 
(c) Ndahiro R et all. This is an open-access a rticle distributed under the terms of the Creative Commons Attribution License, which permits unrestricted use, distribution, and reproduction in any medium, provided the original author and source are properly cited. To view a copy of the license, visit http://creativecommons.org/licens- es/by/4.0/. When linking to this article, please use the following permanent link: http://doi.org/10.24248/EASci-D-20-00015 